\title{
LE NEOCONSTITUTIONNALISME AU BRÉSIL : VERS L'EFFECTIVITE DES DROITS SOCIAUX ET LA LUTTE CONTRE LA CORRUPTION
}

THE NEO-CONSTITUTIONALISM IN BRAZIL: TOWARDS THE EFFECTIVENESS OF SOCIAL RIGHTS AND THE FIGHT AGAINST CORRUPTION

George Sarmento

Professeur à l'Université Fédérale d'Alagoas/FDA. Doctorat en droit public (UFPE). Post-doctorat à l'Université AixMarseille (ISPEC). Chercheur au Laboratoire de droits de l'homme/UFAL george_sarmento@uol.com.br

Résumé : L'effectivité des droits sociaux est le principal défi du constitutionalisme brésilien. Pour le surmonter, il faut combattre la corruption dans la gestion de l'Etat et la crise de confiance. Cette étude consiste à prouver que la loi contre le manquement à l'obligation de probité publique constitue l'un des plus importants instruments légaux pour l'Etat démocratique de droit au Brésil. C'est par la Loi qu'il est possible de rendre viable la bonne gouvernance, de rétablir la confiance populaire dans les institutions d'Etat, d'améliorer la distribution des revenus, de donner davantage d'efficience aux services publics et aux politiques sociales, en plus de sauvegarder la dignité et l'estime de soi de la personne humaine.

Mots-clés: Neoconstitutionalisme - Effectivité des droits sociaux Corruption - Manquement à l'obligation de probité

Abstract: The effectiveness of social rights is the main challenge of the Brazilian constitutionalism. To overcome it we must fight against corruption in public administration and against the crisis of confidence. This research consists in proving that the law against administrative improbity constitutes one of the most important legal instruments for strengthening the Democratic State of Law in Brazil. Through this, is 
possible to make good governance viable, to restore public confidence in the State's institutions, to improve income distribution, to give greater efficiency to public services and social policies in addition to safeguarding the dignity and self-esteem of the human person.

Keywords: Neo-constitutionalism - Effectiveness of social rights Corruption - Administrative improbity

\section{INTRODUCTION}

Après 21 ans de dictature militaire (1964-1985), le Brésil a commencé le processus de démocratisation. En 1988, l'Assemblée nationale constituante a promulgué la nouvelle Constitution de la République fédérative du Brésil, plus connue comme "Constitution citoyenne ». C'était la fin d'un régime autoritaire, antidémocratique, violent, responsable de profondes violations des droits de l'Homme. Le texte constitutionnel était le dépositaire de toutes les espérances du peuple, les consensus possible des forces politiques du pays. Quel est le profil de la nouvelle Constitution ? Quel est son principal fondement axiologique ? Quel est le degré d'effectivité des droits fondamentaux ?

Le constitutionalisme brésilien a été forgé dans un climat de reconstruction nationale, dans l'esprit de la protection des droits de l'Homme et des institutions démocratiques. La Constitution de 1988 représente les efforts de toutes les idéologies politiques pour construire un État démocratique de droit ancré sur des principes axiologiques dotés de force normative et impérative.

Le nouvel ordre constitutionnel privilégie les droits de l'Homme et les libertés publiques. Pour la première fois dans son histoire, le Brésil a assumé la tâche de promouvoir les droits sociaux, tels que la santé publique, l'éducation, le travail, l'habitation, les loisirs, la sécurité sociale, la protection des personnes défavorisées, la protection de la maternité et de l'enfance (art. 6). Parmi ceux-ci, nous pouvons aussi ajouter le droit à l'administration publique probe et transparente (art. 37, caput).

Ces droits sont le produit du libéralisme social adopté par les constituants brésiliens, inspirés du modèle rédistributif où l'égalité d'opportunités, la solidarité et la lutte contre la pauvreté sont les plus importants objectifs de l'État-Providence. Autrement dit, le but de la république est le bien-être de son peuple. Mais celui-ci passe par une bonne utilisation des ressources, par une chasse au gaspillage, parmi d'autres actions publiques. Il exige une bonne redistribution des revenus et une forte présence des services publics (Aubry, 2013) ${ }^{1}$. Bien sûr que le bon fonctionnement de ce système exige l'engagement de la

1 Martine Aubry. Extrait du livre Il est grand temps (Albin Michel), paru en 1997. Cité dans l'introduction du dossier État-Providence : un modèle à réinventer. (Le Monde/Histoire, 2013, p. 5). 
population dans la lutte contre la corruption, puisqu'elle dégrade non seulement l'économie et la gouvernance du pays, mais aussi la qualité des services publiques offerts aux citoyens.

Le modèle de constitutionnalisme adopté par le pays est ancré sur la dignité de la personne humaine comme principe majeur. Sur le plan axiologique, d'autres principes sont dotés de force impérative : la liberté, l'égalité, la solidarité et la démocratie. L'engagement de l'Etat brésilien passe aussi par le pluralisme politique et par la lutte contre toutes les formes de discrimination en raison de la conviction religieuse, politique, philosophique ou raciale. En plus, la Constitution assure la pleine indépendance du pouvoir judiciaire pour juger des affaires relatives au disfonctionnement des politiques publiques dans tous les niveaux de la fédération, y compris les infractions de manquement de probité. On peut affirmer qu'il existe une « dimension politique de la juridiction constitutionnelle ».

\section{Le CONSTItUTIONNALISMe Bresilien : ENTRE LES PRINCIPES ET RÈGLES DE DROIT}

Au Brésil, les droits sociaux sont dotés de force impérative et sont passibles d'application immédiate par le juge. En plus, le juge a le pouvoir de se manifester sur des aspects constitutionnels des politiques publiques, des programmes sociaux et des discriminations positives, sans que cette intervention soit considérée une violation à la séparation des pouvoirs ou du principe démocratique. Tous les citoyens sont sujets des droits sociaux, universels et imposables à l'État. Ils sont titulaires du pouvoir d'exiger des prestations positives, concernant le bien-être de l'individu et de la société. L'effectivité des droits sociaux exige un ensemble de mesures administratives, législatives et judiciaires susceptibles d'assurer des conditions essentielles à la dignité humaine, afin que chaque personne puisse se développer selon ses aspirations et talents (minimum existentiel). Ce sont des droits à des prestations sociales, telles comme l'accès universel à l'enseignement public, au système de santé, aux transports collectifs, à l'emploi etc. (CF, art. 6).

La jurisprudence brésilienne, sous l'influence de la doctrine allemande produite après la Seconde Guerre mondiale, s'est éloignée du positivisme normatif, dont Kelsen est son principal représentant, pour adopter une orientation post-positiviste, selon laquelle l'interprétation de la règle constitutionnelle présuppose le rapprochement entre la morale et le droit. Au lieu de la subsomption, le discours juridique repose sur des arguments rationnels dotés de forte connotation axiologique. Les juges font souvent appel à des principes moraux pour le bien-fondé de leurs décisions. Selon le degré d'abstraction les normes constitutionnelles sont considérées des règles ou des principes, thèse soutenue par Ronald 
Dworkin (2002, p.73) et Robert Alexy (2004, p. 36), parmi d'autres auteurs. Dans le texte constitutionnel, il y a une sorte de cohabitation entre les deux espèces normatives.

Les règles ont un faible degré d'abstraction. Généralement elles ne suscitent aucun doute sur son contenu. C'est la raison pour laquelle leur application est conditionnée à la subsomption d'un fait sous une règle de façon presque mécanique. En revanche, les principes ne sont pas que des normes constitutionnelles ouvertes, porteuses de contenus indéterminés et dotées de grande abstraction en ce qui concerne la prescription normative du droit. Ces aspects exigent un raisonnement particulier du juge lors de l'interprétation des normes constitutionnelles. La pondération est la méthodologie utilisée pour affronter les difficultés herméneutiques et les antinomies entre les principes, assez fréquentes quand il s'agit de l'application des droits fondamentaux. Dans une étude critique sur ce modèle, Pierre Bruné observe que « l'interprétation est donc censée pouvoir parvenir à une solution juste parce que le juge consulte l'univers de la morale et de la philosophie politique. Le juge disposera certes d'un pouvoir discrétionnaire parce que les principes qu'il doit appliquer ne peuvent l'être de façon mécanique et exigent qu'il exerce son jugement prudentiel»(BRUNE, [s.d], p. 4). Nous pouvons présenter deux objections à cette critique. D'abord le juge n'a pas de pouvoir discrétionnaire pour décider selon ses convictions morales, politiques ou idéologiques. Sa subjectivité est très restrictive, puisqu'il a l'obligation de démontrer son raisonnement. Le discours du juge fondé sur des principes doit respecter des critères rationnels Robert Alexy (2004, pp.39-48) en propose trois (pertinence, nécessité et proportionnalité) - et sur des arguments solides afin de convaincre les tribunaux de la conformité de la décision. Ensuite, cette méthodologie d'interprétation est la plus appropriée pour remplir de vide des principes, qui sont des énoncés vagues, ambigus, ouverts et indéterminés. Comment définir de façon générique dignité, solidarité, fonction sociale de la propriété ? Le rôle du juge est de trouver le contenu des principes constitutionnels lors de l'appréciation de certains dossiers, surtout les litiges difficiles, complexes (hard cases).

Pour clarifier cette question, il est possible de s'interroger : comment pouvons-nous distinguer un principe d'une règle ? Plusieurs auteurs se sont consacrés à ce sujet, surtout Robert Alexy et Ronald Dworkin. Mais le concept est très complexe, ce qui présente quelques difficultés théoriques.Il est possible de présenter quelques pistes. D'abord, les principes sont des normes juridiques de droit constitutionnel. Ils présentent une composante axiologique accentuée. Egalement, ce sont des normes indéterminées, vagues, ce qui pose quelques difficultés au niveau de l'interprétation. Finalement, les principes sont des normes fondamentales dans le système juridique. 
Riccardo Guastini(2104, p. 2) présente trois arguments pour justifier la nature fondamentale des principes constitutionnels :

a) " premièrement, il s'agit d'une norme qui caractérise le système juridique en question, en tant qu'élément essentiel à l'identification de la physionomie axiologique du système ;

b) deuxièmement, il s'agit d'une norme qui donne fondement axiologique (une justification éthico-politique) à une pluralité d'autres normes appartenant au même système ;

c) troisièmement, il s'agit d'une norme qui n'exige à son tour aucun fondement, aucune justification éthico-politique, car elle est conçue dans la culture juridique existante comme un axiome, c'est-à-dire une norme évidemment juste et correcte ».

Sur le point de vue théorique, le constitutionalisme brésilien est très attaché à l'effectivité des droits fondamentaux et à la protection du patrimoine public, celle-ci étant essentielle à l'amélioration de l'action publique à tous les niveaux. A partir de la promulgation de la Constitution de 1988, la doctrine et la jurisprudence ont beaucoup évoluées. Parmi les transformations les plus importantes, quelques postulats peuvent être cités: a) La reconnaissance de la force normative de la Constitution ; b) le principe de la progressivité des droits sociaux et la prohibition de la rétroactivité sociale; c) l'élargissement de la juridiction constitutionnelle ; d) la nouvelle herméneutique constitutionnelle ; e) le respect du droit supranational en matière de droits de l'Homme; f) la constitutionnalisation des branches du droit.

\section{LA RECONNAISSANCE DE LA FORCE NORMATIVE DE LA CONSTITUTION}

Le Brésil, comme tous les pays qui ont vécu des dictatures militaires, a adopté une constitution analytique, autrement dit, un texte long et détaillé a fin de se prémunir contre tout risque de retour en arrière. L'introduction des mécanismes de protection des droits fondamentaux ainsi qu'une complexe procédure législative envisagent d'éviter des propositions d'amendements constitutionnels susceptibles de mettre en risque les principaux fondements de la démocratie brésilienne. La rigidité est devenue un véritable rempart pour protéger la stabilité et la pérennité de la Constitution. Les libertés publiques ont été intégrées au noyau dur de la Constitution, insusceptibles d'être supprimées par le Congrès National. Un complexe système de contrôle de constitutionnalité a été conçu sous l'inspiration du modèle nordaméricain et du modèle autrichien : soit le juge le fait dans des dossiers ordinaires où la question est suscitée par l'une des parties du litige, soit le Tribunal Fédéral Suprême, en tant que Cour Constitutionnelle procède à l'analyse de conformité de la loi de façon abstraite.

En plus, les constituants ont confié aux magistrats la tâche 
d'exercer le contrôle de la gestion du patrimoine public dans tous les niveaux de la fédération. Ce phénomène est nommé « la judiciarisation de la politique ", puisque la Constitution assure au Pouvoir Judiciaire la mission de décider sur la validité des politiques publiques dans tous les secteurs des droits sociaux, demander la prestation de comptes aux gouvernants, sanctionner la pratique de la corruption et exiger l'amélioration des services publics.

$\mathrm{Au}$ lieu d'être seulement un texte politique et structurant des institutions de l'État, la Constitution est considérée comme un document normatif, impératif et obligatoire comme n'importe quelle règle juridique. Son efficacité est à la fois verticale et horizontale puisque le juge peut l'appliquer également dans des affaires de droit public et de droit privé.

\section{LE PRINCIPE DE LA PROGRESSIVITE DES DROITS SOCIAUX ET LE PRINCIPE DE LA PROHIBITION DE LA RETROACTIVITE SOCIALE}

L'article $2 \mathrm{du}$ Pacte international des droits économiques, sociaux et culturels, adopté par les Nations Unies du 16 décembre 1966 (entrée en vigueur le 13 janvier 1976, a consacré le principe de la progressivité dans son article 2, par. 1 in verbis :

«Chacun des Etats parties au présent Pacte s'engage à agir, tant par son effort propre que par l'assistance et la coopération internationales, notamment sur le plans économique et technique, au maximum de ses ressources disponibles, en vue d'assurer progressivement l'exercice des droits reconnus dans le présent Pacte par tous les moyens appropriés, y compris en particulier l'adoption des mesures législatives ${ }^{2} \gg$.

Sur ce principe, nous présentons deux remarques :

Pour respecter cette règle impérative de droit international, le Brésil doit prendre toutes les mesures possibles pour faire avancer les politiques publiques en envisageant d'élargir les droits sociaux de façon à bénéficier tous les citoyens. Le Pacte reconnaît que sa mise en œuvre doit être progressive, en respectant les conditions économiques, sociales, politiques et culturelles de chaque pays. La protection sociale exige de gros investissements financiers qui dépendent de la prévision budgétaire et de la planification. Les besoins sociaux augmentent à chaque jour, pendant que les ressources publiques sont limitées. Par conséquent, le gouvernement doit faire des choix difficiles, parfois tragiques en ce qui concerne la distribution sectorielle des dépenses publiques. Mais le principe de la progressivité exige que l'État avance vers l'effectivité des droits sociaux et le bien-être de la population.

Le principe de la progressivité évoque l'idée selon laquelle

2 Le PIDESC a été ratifié par le Brésil par le Décret 591, du 07 juillet 1992. 
l'effectivité des droits sociaux est conditionnée à une certaine gradualité et dépend de plusieurs variables. Il faut reconnaître que les droits sociaux ne peuvent pas être assurés pleinement en un court laps de temps. Mais les États doivent adopter des mesures concrètes qui démontrent son engagement à donner la plus vaste effectivité à ces droits. Dans l'Observation n. 3/90, le Comité DESC souligne que, même si les États démontrent que les ressources sont insuffisantes pour promouvoir certaines obligations sociales, ils doivent s'efforcer « d'assurer l'assurance la plus large possible des droits pertinents dans les circonstances qui lui sont propres ». Evidemment ces droits sont universels : toutes les personnes en sont titulaires. Mais dans un contexte de crise économique, de récession ou de grave pénurie, les gouvernements peuvent adopter des politiques sélectives, pour favoriser les démunis et les groupes vulnérables, lesquels doivent être soutenus par des programmes sociaux.

La deuxième remarque concerne l'interdiction de la rétroactivité sociale. Le législateur, le juge ou le gouvernement ne peuvent pas supprimer certaines prestations sociales essentielles à la dignité de la personne humaine, sans adopter des mesures compensatoires. C'est pour ça que l'Observation 3/90 du Comité DESC affirme que « toute mesure délibérément régressive dans ce domaine doit impérativement être examinée avec le plus grand soin, et pleinement justifiée par référence à la totalité des droits sur lesquels porte le Pacte, et ce en faisant usage de toutes les ressources disponibles ». La règle générale est la réalisation progressive des droits économiques, sociaux et culturels ; l'adoption de mesures régressives est exceptionnelle et impérativement fondées sur des justificatives rationnelles.

\section{L'ELARGISSEMENT DES COMPÉTENCES DE LA JURIDICTION CONSTITUTIONNELLE}

La Constitution de 1988 a renforcé les compétencesdu pouvoir judiciaire et lui a confié, parmi d'autres tâches, le contrôle de constitutionnalité des politiques publiques, des dépenses gouvernementales, des financements de campagnes électorales et, bien sûr, de la sanction des actes de corruption des hauts fonctionnaires et des élus. (articles 101-103).

Pour bien exercer leurs fonctions, ils jouissent de l'autonomie administrative et financière, l'inamovibilité, la nomination à vie et l'irréductibilité de rémunération. Le Tribunal Fédéral Suprême est à la fois une cour constitutionnelle et un tribunal qui a des compétences spécifiques pour juger des affaires en première instance, comme, par exemple, des infractions de droit commun commises par le président de la République, membres du Congrès National et le Procureur général de 
la République (CF, art. 102,b). Les juges de siège, ainsi comme tous les tribunaux brésiliens peuvent se prononcer sur la constitutionnalité des traités internationaux ou d'une loi fédérale. Ils ont la tâche de décider sur la validité d'une loi ou d'un acte de gouvernement local contesté au regard de la Constitution (CF, art. 102, III, a, b, c).

Lorsque le juge analyse un dossier de corruption ou une affaire de manquement au devoir de probité publique, il doit se prononcer tant sur les infractions commises que sur la constitutionnalité de certains actes administratifs comme, par exemple, les dépenses pour la construction d'un hôpital, un contrat de prestation de services, la mise en œuvre d'un programme social, etc. Ainsi, dans le système brésilien, le pouvoir judiciaire est doté de compétences pour décider sur des activités des élus, surtout les membres du gouvernement et leurs auxiliaires (ministres, secrétaires d'État, directeurs des entreprises publiques, etc.).

$\mathrm{La}$ 《 judiciarisation de la politique » est un phénomène du constitutionnalisme contemporain susceptible de tensions et de critiques. Les opposants à tel modèle de constitutionnalisme argumentent que les décisions judiciaires portent atteinte à la séparation des pouvoirs, empêchent l'implantation des programmes proposés par des partis politiques qui sont au pouvoir, bouleversent la démocratie et réduit l'ampleur des décisions discrétionnaires du gouvernement. Il y a, également- ceux qui affirment que ce modèle incite au " gouvernement des juges » et affaiblit les décisions politiques.

Mais le contrôle des politiques publiques, de l'utilisation des ressources budgétaires et du financement des campagnes électorales est aujourd'hui un mécanisme que s'impose dans tous les pays démocratiques. Il est vrai que quelques affaires excessivement médiatisées, donnent parfois l'impression qu'est en cours une campagne de « criminalisation de la politique », menée par des forces occultes. Pourtant l'action ferme de la Police judiciaire (fédérale et civile) et du Ministère public dans la lutte contre la criminalité en col blanc apporte des résultats positifs. Les affaires « Mensalão » et "Opération LavaJato » en sont des exemples paradigmatiques. Il est indiscutable que la condamnation de certains hommes politiques, des fonctionnaires de haut rang et des personnes morales qui ont détourné ou gaspillé le patrimoine public renforceles valeurs républicaines et contribuent à forger une gestion publique responsable, transparente et probe.

\section{LA NOUVELLE HERMENEUTIQUE CONSTITUTIONNELLE}

$\mathrm{Au}$ Brésil, l'interprétation constitutionnelle est devenue une discipline de grand prestige dans plusieurs facultés de droit. Même dans les tribunaux la construction d'un discours juridique rationnel de forte connotation axiologique est un sujet d'actualité. Plusieurs chercheurs 
se consacrent aux études de l'argumentation et de la rhétorique juridique. Les nouvelles méthodes herméneutiques sont conçues pour réduire la subjectivité de juges et favoriser des décisions plus justes et raisonnables

La collision des principes constitutionnels est assez fréquente dans le droit brésilien. Il y a plusieurs situations où des droits fondamentaux du même rang hiérarchique s'entrechoquent. Exemples : liberté de presse et le droit à l'intimité ; la liberté de religion et le droit au repos de voisins ; le droit à la santé et l'insuffisance de ressources budgétaires ; le droit de propriété et la protection à l'environnement, le droit de passation de marchés publics et l'intérêt public. Au lieu de discuter la validité des principes, le juge raisonne en termes de pondération et de prévalence occasionnelle d'un intérêt sur l'autre. L'idée est de les donner la plus vaste applicabilité. C'est pour ça que Robert Alexy affirme que les principes sont des «normes d'optimisation » dont l'effectivité doit être la plus vaste possible devant les circonstances juridiques et sociales (ALEXY, 2004, 37).

A propos de la collision des droits fondamentaux, le juge du Tribunal Fédéral Suprême, Luís Roberto Barroso, argumente qu'il s'agit d'un « phénomène naturel - parce qu'inévitable - dans le constitutionnalisme contemporain. Les Constitutions modernes sont des documents dialectiques qui consacrent des biens juridiques qui s'opposent $\gg$. Pour résoudre ces questions pratiques, les méthodes traditionnelles d'interprétation sont insuffisantes, voire dépassées devant la complexité des sociétés modernes. Pour cette raison, la pondération de normes, de biens et de valeurs est la technique la plus efficace pour rendre les droits fondamentaux effectifs. L'auteur suggère que d'abord les juges doivent faire des concessions réciproques, en préservant au maximum chacun des intérêts en conflit. Et puis « procéder au choix du droit qui devra prévaloir concrètement pour être celui qui réalise de la manière la plus adéquate la volonté constitutionnelle» (BARROSO, 2014).

\section{LA CONSTITUTIONNALISATION DES BRANCHES DU DROIT}

Les Constitutions analytiques ont pour caractéristique principale la densité normative : le texte est long, détaillé, dont l'ampleur abrite plusieurs branches du droit. A tel point qu'aujourd'hui nous pouvons parler de droit civil constitutionnel, droit pénal constitutionnel, droit fiscal constitutionnel et droit administratif constitutionnel. En plus, la Constitution est le fondement de validité des normes de droit infraconstitutionnel. Elle s'impose sur tous les pouvoirs de la République, sans exception. Les valeurs consacrées dans son texte sont porteurs de force impérative dans les relations de droit public et de droit privé. 
La constitutionnalisation du droit est un sujet qui a attiré l'attention de plusieurs juristes français, parmi lesquels Georges Vedel, François Luchaire, Louis Favoreu et Dominique Rousseau. Il a été objet de l'atelier " La constitutionnalisation des branches du droit ", organisé par l'Association Française des Constitutionnalistes en 1996. Au Brésil, ce phénomène est étudié par des importants auteurs, parmi lesquels Ingo Sarlet, Luís Roberto Barroso et Daniel Sarmento. Dans ce contexte, nous citons également l'article fondateur de Paulo Luiz Netto Lôbo, intitulé « La Constitutionnalisation du droit civil » (LÔBO 1999, 99-109), où ce phénomène est abordé dans le cadre du droit privé.

Georges Vedel souligne que le contrôle de constitutionnalité dans plusieurs secteurs des disciplines juridiques a gagné force à partir de 1981, grâce à certaines circonstances politiques et à l'évolution de la jurisprudence. Ce nouveau modèle a suscité des débats acharnés chez les juristes. Pourtant, l'idée que les règles du droit objectif doivent avoir une base constitutionnelle s'impose dans la majorité des pays occidentaux comme, par exemple, en Espagne, Portugal et Allemagne. Le rôle du juge consiste à faire un contrôle de conformité du droit objectif (infra-constitutionnel), ainsi qu'à combler les lacunes légales à partir de l'interprétation des principes constitutionnels (VEDEL, 1996, p.16).Il est aussi le gardien des droits fondamentaux, dont la dignité de la personne humaine $»)$. Devant les règles juridiques ouvertes, ambiguës, génériques et porteuses de concepts indéterminés, le juge constitutionnel est le protagoniste de l'interprétation, puisqu'il fait la médiation entre l'énoncé normatif et la situation concrète qui est l'objet du conflit intersubjectif d'intérêts. Dans certains dossiers, la décision est, par conséquent, le produit d'un complexe exercice herméneutique. Evidemment, les décisions politiques n'échappent pas au contrôle de constitutionnalité, de légalité et de moralité administrative.

L'objectif de cette forme de raisonnement est de construire un « droit vivant » fondé sur l'interprétation consolidée de la règle constitutionnelle ou de la loi. La jurisprudence est sa principale source, mais pas l'unique. Comme observe Thierry de Manno, « ce qui est déterminant dans la formation du droit vivant, ce n'est pas ni rang ni la fonction de l'autorité juridictionnelle qui l'élabore, mais bien le degré de consensus autour d'une interprétation jurisprudentielle de la loi » (DI MANNO, 1996, p.32). Dans le système brésilien, le Tribunal Fédéral Suprême adopte l'interprétation conforme à la Constitution et diffuse des « súmulas vinculantes », qui représentent l'interprétation de la Cour sur des questions controversées. Dotées de force contraignante, elles guident des juges dans les dossiers similaires. De la même façon, les tribunaux sont aussi responsables de construire un cadre jurisprudentiel bien articulé, dont la légitimité est ancrée sur l'accord de la société.

Dans le système brésilien, le juge joue le rôle de protagoniste 
de l'interprétation des principes constitutionnels. Parfois c'est lui qui établit son contenu dans certains dossiers. Pourtant, cet « activisme judiciaire » n'est pas exempt de critiques. Parfois nous reprochons au Pouvoir judiciaire d'usurper la fonction législative et administrative, dans la mesure où les magistrats s'immiscent dans la gestion publique et se prononcent sur la validité des lois démocratiquement votées par le Parlement.

La doctrine française utilise l'expression « gouvernement des juges », depuis 1921, proposée par le juriste Éduard Lambert. Cette notion a été diffusée aux États-Unis - « gouvernment by the judiciary », qu'aujourd' hui utilisent beaucoup plus la notion de « activisme judiciaire ». Au Brésil, cette dernière expression est vastement utilisée dans la littérature juridique.

L'expression gouvernement des juges est polysémique et controversée. Elle est parfois utilisée de façon péjorative et trompeuse. Cette question a fait l'objet d'une importante recherche menée par Michel Troper et Otto Pfersmann. Ils ont essayé de présenter les principales approches sur ce sujet (2001, p. 24-31). Nous pouvons en retenir quelques-unes. Dans l'exercice de leurs compétences,

a) Les juges ont le pouvoir de prendre des décisions politiques pour défendre les intérêts collectifs ;

b) Les juges peuvent affronter des élus pour les obliger à prendre des décisions concernant l'effectivité des droits fondamentaux, y compris les droits sociaux ;

c) Les juges ont le pouvoir de s'autosaisir lors de l'application des principes : c'est la dimension législative de la juridiction ;

d) Les juges ont le pouvoir d'exercer le contrôle de constitutionnalité des lois, des actes administratifs et judiciaires ;

Pour les auteurs, le " gouvernement des juges » est plutôt une expression rhétorique. Il ne s'agit pas de l'appropriation de la fonction législative ou administrative. Le point de départ de leur raisonnement, c'est le propre concept de gouvernement qu'ils présentent : " exerce le gouvernement toute autorité dont les décisions sont susceptibles d'avoir des conséquences pour l'organisation et le fonctionnement de la société » (TROPER, Michel; PFERSMANN, Otto. 2001, p. 33). Or, les décisions judiciaires ont une indiscutable influence sur la vie privée et institutionnelle. Les juges peuvent décider sur les biens ou la liberté d'une personne, quand, par exemple, il se prononce sur un contrat, l'état civil, les droits d'héritage ou de propriété. De la même façon ils ont compétence pour décider sur la qualité des services publics, la validité d'une loi ou la poursuite d'un agent public corrompu. Donc, l'activité judiciaire a une dimension transformatrice de la société, voire de l'Etat constitutionnel de droit.

Par contre, il y a d'autres auteurs qui estiment que le 
gouvernement des juges dans le cadre de l'action politique peut bouleverser l'équilibre entre les pouvoirs de la république et provoquer une crise de légitimité démocratique ${ }^{3}$. Cette peur a été appelée par Jacques Chevalier « le spectre du gouvernement des juges ». Certes, il y a parfois des excès. Mais nous sommes devant une évidence : sans l'intervention du pouvoir judiciaire les réponses à l'accroissement de la corruption seraient faibles, voire inefficaces. Pourtant, la lutte contre la corruption doit respecter des garanties procédurales comme le procès équitable, l'égalité des armes, les droits de défense, la présomption d'innocence, la prohibition des preuves illicites, etc.

Sans doute, la corruption menace l'État du droit et la société démocratique. Mireille Delmas-Marty souligne qu'elle « frappe les droits de l'homme en plein cœur ». C'est la raison pour laquelle la lutte contre la corruption est avant tout une arme politique. Elle reconnait que le gouvernement des juges, ou l'activisme des juges, est essentiel à la protection à la probité publique. A ce propos, l'auteur observe qu'il faut « éviter les excès d'un gouvernement de juges, mais il faut éviter aussi que le spectre du gouvernement des juges ne soit argument pour soumettre les juges au gouvernement». Elle propose une «politique criminelle combinatoire », qui implique la pluralité de moyens et de voies utilisés contre la corruption : réforme des législations nationales pour prévenir, détecter et sanctionner les infractions; renforcement du cadre législatif international ; élargissement de la coopération internationale, parmi d'autres stratégies qui présupposent l'internationalisation de la politique criminelle anticorruption (DELMAS-MARTY, Mireille; MANACORDA, Stefano, 1997. p. 696-700).

Comme le texte constitutionnel brésilien est à l'origine des branches du droit, le juge peut l'appliquer dans toutes les affaires judiciaires, soit de droit public, soit de droit privé. En matière de protection à la probité publique, la Constitution brésilienne a plusieurs règles concernant la responsabilité des agents publics. Selon art. 37, XXI, p. 4, « les actes de malhonnêteté administrative entraînent la suspension des droits politiques, la radiation des cadres de la fonction publique, l'indisponibilité des biens et le dédommagement du Patrimoine public, selon les formes et les degrés établis par la loi, sans préjudice de l'action pénale possible ».

Au Brésil la lutte contre la corruption se déroule en trois fronts : civil, pénal et administratif, comme on l'a déjà exposé. L'indépendance entre l'action civile et l'action pénale adoptée par le système brésilien est un facteur qui prolonge excessivement les procédures et rend la justice plus difficile. Dans le droit pénal français la répression est plutôt criminelle. Quand il s'agit du pacte corrupteur, le droit pénal des

3 Sur ce sujet, nous recommandons la lecture de «Juizes: novo poder», de Rui Verde. 
affaires concilie des sanctions de privatives de libertés et des grosses amendes, infligées à des personnes dépositaires de l'autorité publique, chargées d'une mission de service public ou investies d'un mandat électif. Concernant le crime de corruption active ou passive est un crime puni de peines principales : 10 ans d'emprisonnement et 150.000 euros d'amende (art. 432-11, 433-1, 435-1), mais dans certains cas, comme la corruption passive d'un magistrat en matière criminelle la peine peut atteindre 15 ans de réclusion et 225.000 euros d'amende, plus les peines complémentaires. Pour le trafic d'influence, 10 ans d'emprisonnement et 150.000 euros d'amende. Dans le système français, la Cour de cassation a décidé que la corruption passive " institué principalement en vue de l'intérêt général, tend également à la protection des particuliers ». De cette façon les particuliers, personnes physiques ou morales, qui ont subi des préjudices en raison de l'acte de l'agent public corrompu peut demander une réparation en se constituant en partie civile (VERON, 2011, p. 78).

Concernant les appels d'offre dans des marchés publics, source de plusieurs affaires de corruption, l'article 37, XXI de la Constitution brésilienne est clair :

«Sauf dans les cas spécifiés par la loi, les marchés ayant trait à des travaux, services, achats et aliénations sont adjugés par appels d'offre public garantissant l'égalité de conditions à tous le concurrents et dont les clauses établissent les obligations de paiement ; les propositions retenues sont maintenues, conformément la loi, ce qui n'implique d'autres exigences que celles liées à la qualification technique et économique indispensable à la garantie d'acquittement des obligations $\gg$.

Le constituant brésilien a la préoccupation de protéger les marchés publics des discriminations, du favoritisme, du trafic d'influence et des appropriations abusives. En fait la corruption empêche l'effectivité des droits sociaux, mine la confiance des citoyens au gouvernement et plonge le pays dans la crise de légitimité du pouvoir. C'est la raison pour laquelle il a confié au Ministère Public, à la Cour de Comptes, au Pouvoir judiciaire, parmi d'autres institutions, la tache de mener un combat contre la corruption dans tous les niveaux de la fédération. 


\section{LE RESPECT AU DROIT SUPRANATIONALDANS LA LUTTE CONTRE LA CORRUPTION}

Le Brésil a ratifié toutes les conventions de lutte contre la corruption dans le cadre des Nations Unies, de l'OCDE et de l'OEA.

A partir de l'amendement 45, du 8 décembre 2004, plus connue comme Reforme du Judiciaire, les traités internationaux portant sur la protection des droits de l'homme ont valeur constitutionnelle et sont placés au sommet de l'ordonnancement juridique. Cela implique la possibilité d'application directe des règles de droit international lors du jugement des affaires.

Pour mieux comprendre le contexte brésilien, on mettra en relief l'approche théorique développée par Peter Häberle, l'un de plus imminents représentants du mouvement constitutionnalisme supranational.

En 1978, Peter Häberle a développé le concept de l'Etat Constitutionnel pour identifier l'organisation politique et administrative dont le principe anthropologique et culturel était la dignité de la personne humaine, le respect des droits fondamentaux, la démocratie pluraliste, la séparation des pouvoirs et la souveraineté populaire. Dans ce modèle, la Constitution est le fondement d'un ordre juridique représentatif d'un système de valeurs qui caractérise la société ouverte.

Mais le modèle constitutionnel conçu par Peter Häberle n'est pas autoréférentiel, renfermé sur lui-même. Il propose une organisation ouverte à la fois à l'intérieur et à l'étranger, profondément liée au droit supranational. Quelques années plus tard, il a appelé ce type idéal « État constitutionnel coopératif », cela pour souligner le moment historique où le constitutionnalisme occidental s'engage dans la protection des droits de l'homme, de la paix mondiale et de la responsabilité internationale.

L'une des conséquences naturelles de l'État constitutionnel coopératif est l'intégration entre le droit national et international. Cela implique une ouverture progressive à l'adoption de procédures juridiques communes, l'application interne des traités internationaux. Peter Häberle propose également l'expansion de la juridiction internationale, la coopération entre les pays dans plusieurs domaines, y compris la lutte contre la corruption.

Dans sa formulation théorique, l'harmonisation de la législation nationale au droit supranational est volontaire, puisqu'elle dépend de l'adhésion libre et éclairé de chaque pays, et pas de la force contraignante du droit international. Ainsi, l'État constitutionnel coopératif adopte spontanément des normes crées par la communauté internationale, sans renoncer aux principes éthiques, politiques et philosophiques que lui sont chers.

Le juriste allemand démontre que le constitutionnalisme contemporain subit des transformations importantes dont les résultats ne seront pas perçus qu'à l'avenir. Le droit constitutionnel et le droit 
international ne peuvent plus être considérés comme des structures incommunicables, mais intégrées dans une relation de complémentarité. Grâce à cette action fusionnelle, on peut considérer l'existence d'un «droit communde la coopération ». L'Union européenne, avec son droit communautaire, est actuellement le modèle le plus avancé de la communauté constitutionnelle basée sur la coopération (internationale et régionale) et la solidarité.

Le Brésil suit le même chemin. De plus en plus les tribunaux brésiliens appliquent des règles de droit international, surtout les traités portant sur la protection des droits de homme. Ceux-ci ont une position privilégiée dans l'ordonnancement juridique du pais, puisqu'ils sont considérés comme des règles constitutionnelles, sauf les traités ratifiés avant l'amendement 45/2004 - lesquels, selon l'interprétation du Tribunal suprême fédéral, sont supérieurs aux lois (valeur supra légale). De cette façon, le Brésil adopte le monisme modéré proposé par Peter Háberle, dans la mesure où il avance vers l'harmonisation de la législation nationale et internationale.

\section{LUTTE CONTRE LA CORRUPTION : REFORCEMENT DU CADRE NORMATIF BRÉSILIEN}

Du point de vue de la technique législative, la Constitution de 1988 adopte les règles et les principes comme catégories normatives. Sans abandonner la précision de certaines prescriptions juridiques, la Charte politique se tourne vers la moralisation du droit, ce qui exige du juge la construction d'une axiologie constitutionnelle.

La constitutionnalisation des principes et valeurs par le pouvoir constituant est une autre marque du droit brésilien contemporain. On peut dire que, sous l'influence du droit allemand, la Carte politique est devenue un " ordre objectif de valeurs » Autrement dit, les principes éthiques ont une force normative indiscutable, susceptibles d'être appliqués par le juge Malgré sa nature essentiellement axiologique, les valeurs inscrites dans le texte constitutionnel ont un caractère contraignant et jouent un rôle très important dans le discours et dans l'argumentation juridiques. A ce propos, Stéphane Pierré-Caps remarque que « les valeurs participent ainsi d'une sorte de norme fondamentale posée par la Constitution, autrement dit d'une référence matérielle qui gouverne l'interprétation de l'ordonnancement constitutionnel et, en même temps, en définit aussi la limite» (PIERRÉ-CAPS, [s.d], p. 287).

A partir de son préambule, la Constitution brésilienne considère comme des valeurs suprêmes l'exercice des droits sociaux et individuels, la liberté, la sûreté, le bien-être, le développement, l'égalité et la justice. Elle considère aussi comme principes fondamentaux la citoyenneté, la dignité de la personne humaine, la solidarité et le pluralisme politique, 
parmi d'autres. Le texte privilégie aussi les choix politiques dans le but de permettre le contrôle de l'action politique par le juge. On peut mentionner, par exemple, les directives de l'article 3, qui sont porteuses des valeurs républicaines:

Art. 3. Les objectifs fondamentaux de la République fédérative du Brésil sont les suivants:

I - construire une société libre, juste et solidaire;

II - garantir le développement national;

III - éradiquer la pauvreté et la marginalisation et réduire les inégalités sociales et régionales;

IV - promouvoir le bien de tous, sans préjugés d'origine, de race, de sexe, de couleur, d'âge ou toute autre forme de discrimination.

En plus, la Constitution a autorisé le Pouvoir judiciaire à exercer le contrôle des politiques publiques mises en œuvre par le gouvernement, ainsi que punir le manquement au devoir de probité des hommes politiques et fonctionnaires de haut rang.

$\mathrm{Au}$ Brésil, la lutte contre la corruption se déroule sur trois niveaux indépendants : pénal, civil et administratif.

La structure de la répression pénale commença en 27 février 1967, à l'occasion de la publication du Décret-loi 201. Il prévoit 23 infractions de responsabilité des maires et des conseillers municipaux. Editée pendant le gouvernement militaire, d'origine dictatoriale, il reste encore en vigueur.

Dans le Code pénal, il y a un titre consacré aux attentes contre 1'Administration Publique : corruption active et passive (art. 333 et 317), péculat (art. 312), utilisation indue de finances publiques (art. 315), trafic d'influence (art. 321 et 331), etc.

On peut également mentionner la Loi 7.492, du 16 juin 1986, qui sanctionne les crimes contre le système financier national ; la Loi 9.034, du 3 mai 1995, qui sanctionne les organisations criminelles; la Loi 9.613, du 3 mars 1998, qui sanctionne le blanchiment et occultation de capitaux; la Loi 10.028, du 19 juin 2000, concernant les crimes relatifs au manque de responsabilité fiscale dans la gestion des finances publiques.

Sur le plan civil, plusieurs lois prévoient des sanctions très sévères en ce qui concerne le manque de probité. Nous avons mis l'accent sur la Loi 8.429, qui porte sur les violations contre le devoir de probité. Pourtant, d'autres lois nous permettent d'établir le profil du système de combat contre la corruption dans les pays : Loi 8.666, du 21 juin 1993, qui règlemente les contrats et les appels d'offre dans les marchés publics ; Loi 8.730, du 10 décembre 1993, qui impose aux fonctionnaires publics fédéraux et aux élus le devoir de déclarer les biens et les revenus ; la Loi complémentaire n 101, du 4 mai 2000, concernant la responsabilité des fonctionnaires dans la gestion fiscale.

Sur le plan administratif, la Loi organique de la Cour de comptes 
fédéraux - Loi n. 8.443, du 16 juillet 1992 - apporte plusieurs normes de contrôle externe de la gestion publique dans plusieurs échelons.

\section{Organes Chargés de PRÉVENIR, DÉTECTER ET SANCTIONNER LA CORRUPTION AU BRÉSIL}

Dans la structure administrative et judiciaire brésilienne, plusieurs institutions publiques et privées sont responsables du combat contre la corruption, y compris des associations représentatives de la société civile.

\section{A. Le Ministère Public}

Le Ministère public brésilien joue un rôle très important dans la lutte contre la corruption et le blanchiment de capitaux. Institution indépendante et autonome du point de vue budgétaire et fonctionnel, elle est responsable de la protection du patrimoine publique et de la répression à la criminalité financière, parmi d'autres attributions constitutionnelles. L'organe a deux niveaux d'organisation : Ministère public fédéral et Ministère public des États. Mais le contrôle des activités au niveau national a été confié au Conseil National du Ministère Public (CNMP), dont les Résolutions normatives orientent le travail des procureurs du parquet. Il faut remarquer qu'ici on ne présente qu'une simple synthèse de son fonctionnement, puisque la structure organisationnelle est beaucoup plus complexe.

Dans le cadre de la politique anticorruption, le Ministère Public de chaque État de la fédération a créé des Groupes d'action spéciaux de lutte contre la criminalité organisée, plus connus sous la désignation de GAECO. Ce sont des unités spécialisées qui s'occupent des affaires concernant à la grande corruption, à la criminalité financière, au blanchiment de capitaux et aussi à la coopération internationale ${ }^{4}$.

Envisageant de donner des réponses concrètes contre la corruption, le Ministère public brésilien est responsable de plusieurs affaires, puisqu'il est partie légitime pour demander des requêtes, engager des poursuites et de porter plainte contre des personnes physiques ou juridiques impliquées dans des fautes administratives, l'enrichissement illicite, fraude commise dans le cadre d'une procédure de marché public, financement irrégulier de campagnes électorales, malversation de ressources publics, blanchiment de capitaux, parmi d'autres modalités de corruption lato sensu.

Afin de protéger le patrimoine public, le Parquet peut demander

4 Sur ce sujet, Rapport OCDE de suivi de la mise en œuvre des recommandations au titre de la phase 2. Brésil : phase 2, du 4 juin 2010. Disponible sur le site : $<$ www.oecd.org/fr/france $>$. Accès : 10 dec. 2014. 
au juge des mesures préliminaires et conservatoires comme, par exemple, l'interception des communications téléphoniques et télématiques, la levée du secret bancaire, fiscal et patrimonial du prévenu, le gel des biens et de valeurs, gestion des avoirs, etc. En cas de condamnation civile, les sanctions sont très sévères : perte de la fonction publique, perte des avoirs, amendes pécuniaires, suspension des droits politiques, restitution des avoirs obtenus irrégulièrement, l'interdiction de participer à des procédures de marché public. Si la condamnation est aussi pénale, le juge imposera au prévenu une peine de prison et d'autres sanctions accessoires.

La Loi 12.850, du 2 aout 2013, concernant la lutte contre les organisations criminelles, permet au Ministère public la signature d'un accord de collaboration avec le prévenu, lequel pourra bénéficier d'une réduction de la peine, voire du " pardon judiciaire », à condition qu'il apporte des informations susceptibles de sanctionner les membres du réseau criminel. Le collaborateur - aussi nommé délateur par la presse et chez les avocats - bénéficie également d'autres avantages procéduraux comme, par exemple, la préservation de son identité et l'acceptation dans le « Programme fédéral d'assistance des victimes et des témoins menacés ${ }^{5}$ ».

\section{B. Le Conseil de contrôle des activités financières}

La lutte contre la délinquance financière et la corruption a été renforcée par la création du Conseil de contrôle des activités financières qui s'occupe de la prévention et de la détection des crimes de blanchiment de capitaux et financement du terrorisme. Crée par la Loi 9.613, du 3 mars 1998, cet organe est responsable de la coordination de la coopération nationale et internationale et fonctionne aussi comme lanceur d'alerte auprès des autorités brésiliennes.

Le signalement du non-respect est un outil essentiel dans le cadre de la répression du blanchiment de capitaux et de la grande corruption. Il permet des enquêtes sur des opérations financières suspectes et combat l'opacité de certaines transactions dans le monde des affaires. Mais son efficacité est conditionnée au renforcement du contrôle interne et de l'élargissement de la coopération judiciaire internationale.

Le COAF est aussi responsable de la formation des fonctionnaires publics et des dirigeants des entreprises privées, y compris les institutions financières et le Ministère public. Dans le cadre du Programme national de renforcement des moyens d'action et de formation en matière de lutte contre la corruption et le blanchiment de capitaux(PNLD), lancé par le Ministère de la Justice, le COAF a formé, entre 2004 et 2009, 6.180 professionnels, qui ont obtenu le certificat d'aptitude à la prévention et à la lutte contre le blanchiment de capitaux

5 Loi 9.807, du 13 juillet 1999. 
ou le certificat de spécialisation dans la prévention et la lutte contre le blanchiment de capitaux, selon leurs compétences ${ }^{6}$.

Plusieurs mesures ont été adoptées par le gouvernement brésilien pour contrôler les activités financières des personnes politiquement exposées, afin de prévenir et de sanctionner la pratique de blanchiment de capitaux. L'article 3o-B de l'Instruction normative de la Commission des Valeurs Mobilières (CNM) a donné une très grande ampleur au concept de PPE et a imposé aux institutions financières le renforcement des mesures d'identification, de signalement d'opérations suspectes et de conservation des informations de leurs clients.

\section{Le contrôle général de l'Union}

Le Contrôle général de l'Union (CGU) est l'organe responsable pour orienter le Président de la République sur des questions relatives à la protection du patrimoine public et la transparence dans la gestion publique. Il a aussi pour tâche le contrôle interne et la prévention de la corruption. Toutes ses compétences sont prévues dans la Loi n. 10.683, du 28 mai 2003 et du Décret n 8.109, du 17 septembre 2013.

Dans le cadre de la prévention de la corruption transnationale, la CGU s'est chargée de la diffusion de la Convention de l'OCDE auprès des plus gros exportateurs brésiliens, rappelant leurs obligations envers leurs obligations dans le commerce international. Elle invite aussi les entreprises à adopter des principes d'intégrité et de codes de bonnes pratiques, en les sensibilisant à rejeter le paiement de pots-de-vin à des agents public nationaux ou étrangers.

Enfin, la CGU est responsable de plusieurs initiatives comme, par exemple, l'adoption de critères susceptibles de permettre l'élaboration d'une « Liste des entreprises vertueuses », la publication du manuel « Responsabilité sociales des entreprises dans la lutte contre la corruption », partenariat avec l'institut Ethos envisageant la diffusion d'un pacte pour l'intégrité contre la corruption ${ }^{7}$.

\section{L'EFFECTIVITE DU DROIT A L'ADMINISTRATION PUBLIQUE PROBE ET EFFICIENTE}

Selon l'OCDE, en 2014, le Brésil a comblé les lacunes juridiques en matière de corruption internationale, mais l'institution espère que ces mesures vont se traduire par une action répressive efficace et intensifié. L'avancement le plus important de cette période a été la publication de

6 Loi 9.807, du 13 juillet 1999.

7 Sur ce sujet, Rapport OCDE de suivi de la mise en œuvre des recommandations au titre de

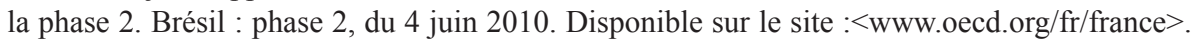
Accès : 15 dec. 2014. 
la nouvelle loi sur la responsabilité des entreprises. Pourtant la faible effectivité de son cadre législatif est encore une source de préoccupation.

Dans le rapport du Groupe de Travail de l'OCDE sur la corruption transnationale, publié en 2014, on trouve des importantes recommandations concernant la politique criminelle brésilienne anticorruption :

«1. D'être plus proactif en matière de détection, d'enquête et de poursuite de la corruption transnationale ;

2. de promulguer instamment le décret d'application annoncé de sa Loi sur la responsabilité des entreprises ;

3. de clarifier sa nouvelle loi sur la responsabilité des entreprises, notamment sur la procédure visant à établir la responsabilité et à imposer des sanctions, afin de tirer pleinement bénéfice de cette législation ;

4. d'assurer un suivi de l'arsenal, renforcé des mesures dont disposent les autorités brésiliennes pour encourager le signalement et la divulgation d'actes de corruption transnationale - notamment par le biais d'accords de coopération et de clémence avec des individus et des entreprises ;

5. De continuer à encourager les entreprises, notamment les PME, à mettre au point et à adopter des systèmes de contrôle interne, de déontologie et de conformité adéquats afin de prévenir et de détecter la corruption transnationale.

6. D'adopter des mesures de protection complète des lanceurs d'alerte afin de protéger les salariés du secteur privé dénonçant de faits de corruption transnationale ${ }^{8} \gg$.

Or, les résultats diffusés par l'OCDE nous permettent de faire trois remarques. D'abord, il est perceptible une importante évolution du cadre législatif brésilien. Ensuite, les entreprises et les institutions financières doivent s'engager dans la lutte contre la corruption transnationale. Enfin, le manque d'effectivité concernant l'application des lois reste encore un problème majeur dans cette démarche.

Premièrement, il est clair que depuis 1992 le pays a mis en œuvre une importante production législative pour affronter la corruption dans tous les niveaux de la république. La répression a été renforcée au niveau civil comme au niveau pénal. Les mécanismes de contrôle sont aussi plus rigides qu'avant. Donc, sur le plan législatif, le droit brésilien s'harmonise aux conventions internationales, ce qui représente un progrès dans la voie de la protection à la probité administrative.

Deuxièmement, la lutte contre la corruption concerne aussi d'autres acteurs, y compris les entreprises et les banques. L'OCDE, met en relief le devoir du gouvernement brésilien d'encourager les entreprises à élaborer des codes déontologiques, à adopter des

8 Phase 3 : Report on implementing the OECD anti-bribery Convention in Brazil. 2014. Disponible sur : <www.oecd.org>. Accès : 15 dec. 2014. 
pratiques de bonne gouvernance, de créer des systèmes de contrôle internes, de signaler des opérations suspectes. La bonne formation des cadres et la probité dans des relations commerciales sont des facteurs incontournables pour assurer l'égalité d'opportunités dans l'occupation des marchés publics.

Mais le principal obstacle à ce projet est la faible effectivité des lois anticorruption. L'OCDE insiste sur la nécessité de développer des mécanismes de prévention plus efficaces, en adoptant une posture proactive. Autrement dit, 1'Etat doit s'attaquer à l'origine de la corruption afin d'en restreindre au maximum sa pratique. De l'autre côté, l'application des sanctions doit être plus rapide, tout en respectant les droits de défense. La lenteur des réponses judiciaires mine la confiance que les citoyens déposent dans des institutions démocratiques. En conséquence, le grand défi du pays repose sur l'effectivité du droit à l'administration publique probe et efficiente à partir d'un ensemble de mesures prévention, détection et sanction de la corruption politique. Cette tâche est difficile dans la mesure où le pouvoir judiciaire, le Ministère public et même l'administration publique ne sont pas bien structurés pour affronter la croissante complexité du crime organisé, qui bénéficie de la dérèglementation des marchés financiers internationaux et de la complicité de ce qu'on appelle " la bourgeoisie mafieuse " des avocats, comptables, banquiers, consultants, fonctionnaires de haut rang. En fait, « l'interpénétration de la criminalité et du monde politique permet à celle-ci à la fois d'accéder aux marchés publics et d'obtenir une protection à l'échelle nationale (démantèlement de cellules judiciaires jugées trop efficaces, comme en Italie) et internationale (adoption de normes peu contraignantes $)^{9} \gg$.

Les électeurs ont aussi un rôle très important à jouer. Le jugement politique est essentiel pour renforcer la lutte contre la corruption. Un exemple, s'impose. $40 \%$ des sénateurs et députés fédéraux brésiliens élus en 2014 sont objet de poursuites judiciaires, la plupart en raison des crimes de corruption (SASSINE; BRESCIANI; SOUZA, 2014). Cette posture de l'électorat, pleine de condescendance, renforce le sentiment d'impunité et mine la démocratie. La tolérance de l'électeur à l'égard des atteintes à la probité publique est au centre des recherches développées par Pierre Lascoumes. Une grande partie de la population se méfie des hommes politiques, surtout de ceux qui sont impliqués dans des scandales, vastement diffusés par les médias. Cependant, les sanctions politiques sont faibles, voire inexistantes. Selon l'auteur, trois facteurs expliquent ce paradoxe : « tout d'abord, ce qui fait corruption pour les uns ne le fait pas pour les autres. Les significations données à ce terme sont très diverses. Ensuite cette forme de déviance bénéficie

9 L'Atlas du monde diplomatique 2014-mondes émergents. La mondialisation criminelle, p.64. 
d'un vaste ensemble de justifications et d'excuses qui en relativisent la gravité. Enfin, il existe des liens étroits entre le type de perception de la corruption et la perception du politique » (LASCOUMES, 2011, p. 17). Mais le phénomène est tellement complexe qu'on peut ajouter d'autres explications. Quand il s'agit des pays en voie de développement, comme le Brésil, l'opinion publique est sidérée par l'image du candidat soigneusement retouchée par le marketing politique. Ensuite, le financement privé de campagnes électorales renforce le " pouvoir de persuasion » des grands partis politiques envers l'électorat grâce à dépenses pharamineuses. De l'autre côté les promesses des candidats alimentent l'espoir des communautés plus défavorisés et susceptibles de se laisser convaincre par le clientélisme et les tromperies les plus variées. Enfin, on considère que le refus des citoyens honnêtes de participer à la vie politique laisse un vide qui est rempli par des criminels, surtout dans les parlements et dans la gestion publique. Cet ensemble d'éléments explique les distorsions de la démocratie et la crise de la représentation politique, ainsi que le manque de confiance de citoyens envers les élus.

\section{Conclusions}

Phénomène mondial, la corruption affecte la gestion publique et l'économie des nations contemporaines. Les pays se situant à la périphérie du capitalisme sont les principales victimes de ce fléau et les conséquences sont manifestes : la qualité de vie se dégrade, la population s'appauvrit, la violence croît, les droits de l'homme sont systématiquement bafoués, les politiques publiques inefficaces et les taux d'intérêt internationaux augmentent. Les pays riches aussi en sont affectés. Le blanchiment d'argent, la criminalité financière, les fraudes dans les appels d'offre et le financement privé des campagnes électorales sont responsables de la circulation clandestine de milliards de dollars tous les ans. En fait, la corruption s'est mondialisée. Elle ne respecte aucune frontière, aucun peuple, aucune idéologie. La combattre est donc devenu l'une des plus grandes priorités de la communauté internationale.

Le Brésil fait partie de ce projet. Bien que les indices de corruption dans le pays soient alarmants, plusieurs mesures ont été prises dans le sens de réduire de manière significative les actes de corruption dans le cadre de ses organismes administratifs.

La Loi de 8.429/92 a renforcé l'engagement dans la probité, dans la bonne gestion et dans le respect des principes de l'administration publique. La corruption a été affrontée par de rigoureuses sanctions qui se projettent dans la dimension patrimoniale et politique de l'agent malhonnête. Son application croissante par le Judiciaire tend à̀ décourager les pratiques telles que l'enrichissement illicite, l'usage abusif des ressources financières, le trafic d'influence, le népotisme et 
la déloyauté envers les institutions. Ce qui est vraiment nouveau c'est que, de nos jours, le fonctionnaire a conscience de la responsabilité qui entoure l'exercice de son poste public : se laisser séduire par la tentation du pot-de-vin est une conduite trop risquée, au gain douteux, pouvant le mener irrémédiablement à la ruine et à la perte de sa citoyenneté.

A partir de cette loi, le Brésil a ouvert ses portes à la mise en place de l'administration publique gestionnaire, fondée sur la professionnalisation de ses fonctionnaires, sur l'autonomie, l'impersonnalité, l'efficacité et la tutelle des intérêts collectifs. L'usage rationnel des ressources publics et le respect des principes déontologiques de la fonction administrative vont répercuter sur l'amélioration des services publics et sur la qualité de vie de la population, engendrant ainsi développement et harmonie dans le pays.

Outre cet aspect, la baisse de la corruption au Brésil ne sera perceptible qu'à partir de changements structuraux dans le fonctionnement du système administratif, qui, comme nous l'avons soutenu précédemment, suppose au préalable 1) la démocratisation des processus de décisions quant à l'allocation des ressources publiques, 2) la transparence des appels d'offre, 3) l'indépendance du Pouvoir judiciaire, 4) le renforcement des organismes de contrôle financier et comptable, 5) la mobilisation de la société civile en faveur du Trésor public et de la bonne qualité des services publics, 6) la liberté d'exercice dans l'investigation journalistique, 7) la formation permanente des fonctionnaires, 8) le financement public des campagnes électorales, 9) la formation et l'articulation des juges, des procureurs de la République, des autorités du fisc et de la police dans le combat contre la corruption et 10) le respect des droits fondamentaux.

Il y a plus. Il ne suffit pas que l'agent public soit honnête. Encore faut-il qu'il ait une préparation adéquate à l'exercice de la fonction publique et qu'il prenne l'engagement de faire bon usage des ressources de l'Etat et d'améliorer les services publics. De plus, le comportement des fonctionnaires doit être en harmonie avec les principes de l'administration inscrits dans la Constitution fédérale. C'est dans l'Ethique administrative que la fonction publique va trouver l'ensemble des valeurs de base pour la construction d'une bureaucratie efficace tournée vers le bien commun.

La réalisation des objectifs de l'Entreprise-Etat dépend du bon fonctionnement de sa machine administrative. Autrement dit, l'application des fonds publics doit êtretournée vers les «bénéfices collectifs ». La corruption appauvrit l'Etat dans la mesure où elle vise à la promotion de l'appropriation privée du patrimoine public, empêchant de la sorte la distribution de la justice sociale, l'amélioration des services publics et le développement du pays. Ceci explique pourquoi la lutte contre la corruption est l'une des conditions préalables les plus importantes pour la modernisation de 1'Etat. Sans cela, la nation subira 
les effets de la pauvreté, de la crise de légitimité des institutions, de la concentration des revenus, de l'immobilisme social et financier.

Nous pouvons ainsi montrer les deux axes d'action pour contenir l'escalade de corruption au Brésil. Le premier réside dans l'engagement réel de la société civile dans le combat contre toutes les formes de corruption, surtout celles du clientélisme, du népotisme et de la vénalité du fonctionnariat. Tous les efforts doivent être canalisés dans l'inspection de l'usage des fonds du trésor public, en constituant un véritable réseau de vigilance des actes pratiques par les fonctionnaires. La démocratie participative offre des mécanismes d'organisation sociale capables d'assurer le succès de cette stratégie. L'engagement civil et communautaire renforcera le système de contrôle des dépenses publiques et évitera le détournement ou l'utilisation indue des biens collectifs.

Le deuxième axe passe par la reconnaissance de l'importance jouée par les lois dans le combat contre la corruption. Tout pays soucieux d'en finir avec les crimes en « col blanc » doit commencer par se doter d'un cadre normatif capable de faire front à la corruption. Le vide légal crée un rideau d'impunité qui affaiblit l'Etat et plonge la société dans l'anomie la plus profonde. Au cours des années 90, le Brésil a élaboré une législation capable de réprimer les conduites malhonnêtes, de moraliser le processus d'appel d'offre et d'implanter une gestion fiscale efficace. Ce processus continue aujourd'hui avec des réformes de la législation pénale.

Cependant, la tutelle administrative sur le patrimoine public n'atteindra sa dimension maximale qu'avec l'application de normes juridiques qui agissent sur l'usage rationnel des montants budgétaires, sur la transparence dans l'application des fonds d'Etat et sur la clarté des appels d'offre. Ceci ouvre un champ de recherche très vaste dans des domaines spécifiques du Droit administratif et du droit pénal encore peu explorés dans les études avancées. En ce qui nous concerne, nous nous contentons de montrer la tâche qui incombe à la Loi d'improbité pour ce qui est du renforcement des bases dans la gestion administrative moderne, en particulier par rapport à̀ la punition des fonctionnaires corrompus.

$\mathrm{Du}$ succès de cette mobilisation internationale contre la corruption dépend le développement de beaucoup de pays périphériques. On estime que 600 milliards de dollars circulent annuellement de façon clandestine dans le marché financier mondial, échappant au paiement d'impôts. Une grande partie de cette fortune provient d'actes de corruption, principalement de subornation payée à des fonctionnaires jouissant d'un pouvoir de décision. L'argent sale ne respecte aucune frontière, aucune souveraineté, aucun système politique ou économique. Le crime organisé s'infiltre dans toutes les formes de gouvernement et ronge la crédibilité des institutions démocratiques. Isolement, aucune nation n'est en mesure d'éradiquer la criminalité financière. C'est là la 
tâche de la coopération internationale qui réclame la réunion de toutes les forces pour la vaincre définitivement.

A l'autre bout de la corruption, se trouvent les entreprises transnationales ou nationales de grande envergure qui font du paiement de pots-de-vin une stratégie pour la conquête de marchés publics. Pour parvenir à leurs fins, elles financent des campagnes électorales, font des dons généreux, versent de juteux montants pour la publicité aux organismes de communication, participent à des actions philanthropiques, influencent l'élaboration des budgets; tout cela pour obtenir des privilèges à la signature de contrats avec l'Etat. En gros, elles agissent par le truchement de stratagèmes tels que le trafic d'influence, le paiement de « commissions » occultes, la surfacturation de travaux et services, et la séduction de groupes politiques.

Plus que jamais, l'Etat doit assumer son rôle de promotion du bien-être social. Cela n'est possible qu'avec une gestion responsable des fonds publics, la démocratisation des services publics essentiels et l'infrastructure éthique des actions administratives. Se battre contre le détournement des montants budgétaires, veiller à la clarté des licitations, qualifier constamment les cadres du service public, exonérer les agents publics malhonnêtes et promouvoir la satisfaction des intérêts collectifs, telles sont les stratégies qui doivent être adoptées par les gouvernants intéressés par la réalisation concrète de la légalité et de la moralité en tant que valeurs suprêmes d'un régime démocratique.

Il est essentiel, aussi, que l'Etat soit en mesure de se doter d'un appareil capable de réagir énergiquement aux assauts du crime organisé. La formation de forces d'action composées de juges, de membres du Ministère public, de policiers, de comptables et d'agents du fisc a donné de bons résultats ici et à l'étranger. Le travail intègré dans les institutions a l'avantage de prendre les devants sur les évènements, d'élargir le spectre des investigations et d'atteindre des résultats en un temps moindre. Il est temps que le Brésil abandonne sa posture réactive et entreprenne la prévention contre les crimes en « cols blancs ». Cela sera possible moyennant la modernisation de la législation pénale, la mise en place d'un service d'intelligence agissant au niveau national et international, la création de commissariats spécialisés dans les crimes contre l'administration publique et le blanchiment d'argent.

Hors de ces engagements, le Brésil ne pourra guère trouver la voie du développement durable, de l'inclusion sociale, du bonheur de son peuple et de la solidité du régime démocratique. L'éradication de la corruption s'impose comme l'une des priorités nationales, le seul espoir de s'assurer la jouissance des droits de l'homme dans toute sa plénitude. Tout cela justifie l'existence du droit fondamental à l'administration publique probe et efficiente dans la Constitution brésilienne. 


\section{REFERENCES}

ALEXY, Robert. Epílogo a la teoría de los derechos fundamentales. Madrid: Peritia Iuris, 2004.

BARROSO, Luís Roberto. Le neoconstitutionnalisme et la constitutionnalisation du droit. Disponible : $<$ www.luisrobertobarroso. com.br>. Accès : 15 de. 2014.

BRUNE, Pierre. Analyse critique du constitutionnalisme contemporain. In : Droit et Economie. Interférences et interactions, Mélanges en l'honneur de Michel Bazex. Paris : Litec, Lex-Nexis, p. 4.

DELMAS-MARTY, Mireille; MANACORDA, Stefano. La corruption : un défi pour l'État de droit et la société démocratique. In : Revue de Sciences Criminelles, juillet-septembre, 1997. P. 696-700.

DI MANNO, Thierry. Réserves d'interprétation et «droit vivant». In : La constitutionnalisation des branches du droit. Aix-en-Provence : Economica - Presses universitaires d'Aix-Marseille, 1996, p.32.

DWORKIN, Ronald. Levando os direitos a sério. São Paulo: Martins Fontes 2002.

GUASTINI, Riccardo. Les principes de droit en tant que source de perplexité théorique. Communication présenté au colloque «Les principes en droit ». Université Jean Moulin, Lyon, décembre 2007. Disponible : <www.guiri.it>. Accès : 10 dec. 2104, p. 2.

HÁBERLE, Peter. El Estado Constitucional. México : Universidad Autónoma de México, 2003.

LASCOUMES, Pierre. Une démocratie corruptible : arrangement, favoritisme et conflit d'intérêts. Paris : Seuil, 2011, p. 17.

LÔBO, Paulo Luiz Netto. Constitucionalização do Direito Civil. In : Revista de Informação Legislativa. Brasília : jan/mar 1999.

OCDE. RapportOCDE de suivi de la mise en œuvre des recommandations au titre de la phase 2. Brésil : phase 2, du 4 juin 2010. Disponible sur le site : <www.oecd.org/fr/france>. Accès : 10 dec. 2014.

. RapportOCDE de suividelamise en $œ u v r e$ desrecommandations au titre de la phase 2. Brésil : phase 2, du 4 juin 2010. Disponible sur le site : <www.oecd.org/fr/france $>$. Accès : 15 dec. 2014.

. Report on implementing the OECD anti-bribery Convention in Brazil. 2014. Disponible sur : <www.oecd.org>. Accès : 15 dec. 2014. PIERRÉ-CAPS, Stéphane. La Constitution comme ordre de valeurs. In : La Constitution et les valeurs - Mélanges en l'honneur de 
Dmitri Georges Lavroff. Paris : Dalloz, p. 287.

SASSINE, Vinicius ; BRESCIANI, Eduardo ; SOUZA, André. Congresso: quase $40 \%$ dos parlamentares eleitos são investigados pela justiça. O Globo, le 12 octobre 2014. Disponible : <www.oglobo. oglobo.com>. Accès : 15 dec. 2014.

TROPER, Michel; PFERSMANN, Otto. Existe-il un concept de gouvernement des juges? In : Gouvernement des juges et démocratie. Paris : Publications de la Sorbonne, 2001, p. 24-31.

VEDEL, Georges. Propos d'ouverture. In : La constitutionnalisation des branches du droit. Aix-en-Provence : Economica - Presses universitaires d'Aix-Marseille, 1996, p.16.

VERON, Michel. Droit Pénal des Affaires. Paris : Dalloz, 2011, p. 78. 